Check for updates

Cite this: DOI: 10.1039/c8nj03548b

\title{
Gaining insight into the photophysical properties of a coumarin STP ester with potential for bioconjugation $\uparrow$
}

\author{
M. González-Pérez, (D) a S. Y. Ooi, ${ }^{b}$ S. Martins, ${ }^{a}$ João P. Prates Ramalho, (iD b \\ A. Pereira ${ }^{a b}$ and A. T. Caldeira (iD *ab
}

\begin{abstract}
The photophysical properties of a coumarin 392 4-sulfotetrafluorophenyl ester, C392STP (sodium (E/Z)4-(4-(2-(6,7-dimethoxycoumarin-3-yl)vinyl)-benzoyl)-2,3,5,6-tetrafluoro-benzenesulfonate), an amine reactive coumarin with potential for bioconjungation, have been studied in different solvents. When increasing the solvent polarity, strong red shifts (31 to $56 \mathrm{~nm}$ on going from dioxane to DMSO and PBS, respectively) were observed in the emission spectra while there were minor changes in the absorption spectra. It was found that in addition to non-specific solute-solvent interactions, in protic solvents specific interactions such as hydrogen-bonding occurred. The photophysical behaviour of coumarin also pointed to an increase of the polarity of the molecule upon excitation. The molar absorption coefficient, $\varepsilon_{385}=1.03 \times 10^{4} \mathrm{M}^{-1} \mathrm{~cm}^{-1}$, and brightness, $\varepsilon \times \Phi=1.18 \times 10^{3} \mathrm{M}^{-1} \mathrm{~cm}^{-1}$, of the coumarin ester were found to be moderate in PBS even if the fluorescence quantum yield ( $\Phi=0.115$ ) was found to be relatively low. The large Stokes shifts $\left(\Delta \lambda=\lambda_{\mathrm{em}}-\lambda_{\text {abs }}\right.$ was found to increase from $74 \mathrm{~nm}$ in dioxane to $136 \mathrm{~nm}$ in PBS), photostability and pH insensitivity are characteristics that turn C392STP into a promising fluorescent dye with potential applications in different fields.
\end{abstract}

Received 16th July 2018 ,
Accepted 26th August 2018

DOI: $10.1039 / c 8 n j 03548 b$

rsc.li/njc density, fluorescent chemosensors, biosensors and as dyes in fluorescent adhesives for artifact reconstruction repair in art conservation. ${ }^{1,7,12-15}$ The applications of fluorescent dyes are highly dependent on their photophysical and photochemical properties - UV-vis absorption and fluorescence spectra, molar extinction coefficients, quantum efficiencies, Stokes shifts, $\mathrm{pH}^{-}$and thermal stabilities among others ${ }^{16,17}$ - and on their variation with the surrounding medium. ${ }^{13,18,19}$ Studying the properties of newly synthesized fluorophores is of utmost importance for determining their potential applications and for better exploiting their characteristics.

Thus, the determination of the photophysical properties of coumarin derivatives has been and continues to be the focus of numerous investigations. ${ }^{2,16,20}$ The fluorescent coumarins used as dyes and sensors have excellent spectroscopic properties such as high quantum yields (up to 0.90), good extinction coefficients (10000-40000), and large Stokes shifts (up to $160 \mathrm{~nm}$ ). ${ }^{4,21}$ New coumarin-based fluorophores with improved Stokes shifts, high fluorescence quantum yields and/or molar absorption coefficients are continuously being synthesized. ${ }^{3,6,13}$ In fact, various substituted coumarins (and hybrid dyes based on them) are among the most applied and important fluorophores exhibiting large Stokes shifts. ${ }^{6,12}$

In this work, the spectral characteristics of a recently synthesized coumarin 4-sulfotetrafluorophenyl ester (C392STP, Scheme 1) 
were studied. $^{22}$ This compound has been previously shown to be an efficient building block for synthesizing RNA-FISH probes by labelling of amine-modified oligonucleotides. ${ }^{22}$

Systematic analysis of solvent effects on its electronic absorption and emission spectra was carried out using several solvatochromic models (Lippert-Mataga, Bakhshiev, Ravi, Kawski-ChammaViallet, Kamlet-Taft and Catalan). ${ }^{23-25}$ Also, the photostability, $E / Z$ thermoisomerization and the influence of $\mathrm{pH}$ on the stability of the compound in aqueous solution were investigated.

To the best of our knowledge, this is the first study focused on gaining insight into the photophysical properties of this dye and their comparison with those of other coumarins and commercially available fluorescent compounds. This could open the door for elucidating its potential as a fluorescent dye in diverse fields.

\section{Results and discussion}

The coumarin 392 STP ester resulting from the synthesis performed by us, ${ }^{22}$ with a high yield (98\%), is formed as a mixture of $E$ and $Z$ isomers (Scheme 1 ). The $E / Z$ ratio determined by H-NMR (84:16) suggested the existence of a small energy gap between the $E$ and $Z$ species and the higher stability of the $E$ isomer.

\section{Spectral characteristics of C392STP and solvent effects}

C392STP is a yellow powder (Fig. 1A) that has been revealed to be a promising fluorescent dye for use in the RNA-FISH technique. ${ }^{22}$ Its solutions in polar protic and aprotic solvents are yellow under daylight illumination (Fig. 1B).

Despite the proven potential of C392STP as a fluorescent marker, its photophysical properties have not yet been investigated in depth. It is crucial to deepen the knowledge about the photophysical characteristics of this fluorophore and its interaction with solvents, not only for improving its performance as a fluorescent label for biomolecules but also for discovering new potential uses for it. The influence of environmental effects on the photophysical properties of substituted coumarins has already been extensively studied with in silico calculations and steady state and time resolved spectroscopy. ${ }^{2,26-31}$ It has been found that the variation of the surrounding medium (in terms of polarity, dielectric constant, polarizability) affects the ground and excited states differently. ${ }^{13,27,29,32}$ The absorption and emission spectra of these compounds can be influenced in such a manner by the solvent that the solvatochromism observed for

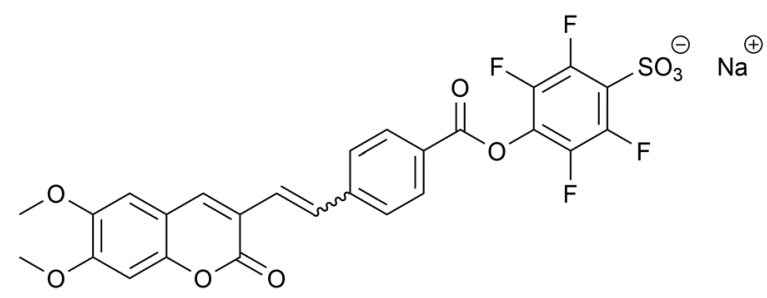

Scheme 1 Structure of C392STP, sodium (E/Z)-4-(4-(2-(6,7-dimethoxycoumarin-3-yl)vinyl)-benzoyl)-2,3,5,6-tetrafluoro-benzenesulfonate.

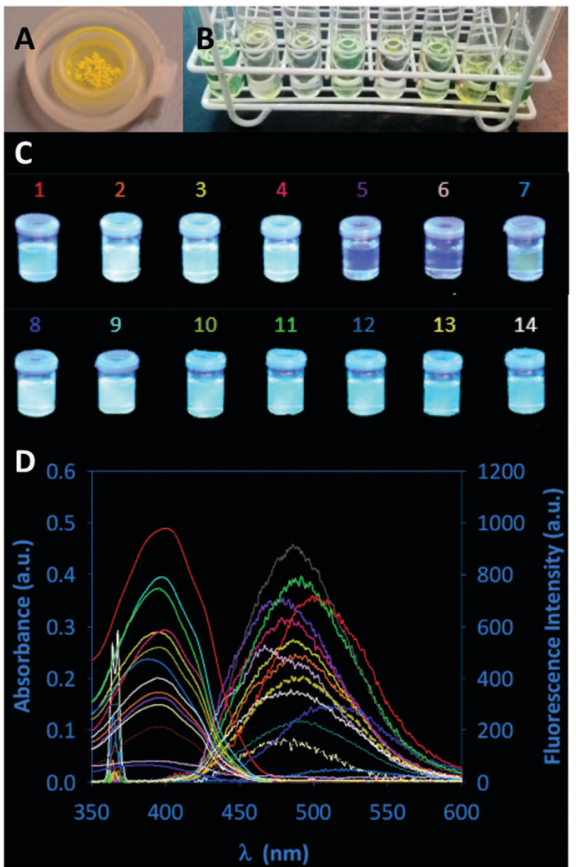

Fig. 1 C392STP powder (A), and C392STP solutions under daylight illumination $(B)$ and under UV illumination $(365 \mathrm{~nm})$ in different solvents: DMSO (1), DMF (2), acetone (3), THF (4), ethyl acetate (5), dioxane (6), PBS 22 mM (7), water (8), ethanol/water 40/60 (v/v) (9), ethanol/water 20/80 (v/v) (10), methanol (11), ethanol (12), propanol (13), 1-butanol (14) (C). Absorption and emission spectra of C392STP obtained in solvents 1-14 (D).

some coumarins has previously been exploited for monitoring the polarity and microviscosity of the environment ${ }^{3,15,32,33}$ and for determining the dipole moments of the ground and excited states as well as the variation between them. ${ }^{13,27,30}$

\section{In silico calculations}

It is known that Density Functional Theory (DFT) methods can predict with good accuracy the structure and spectroscopic properties of coumarins. ${ }^{21}$ Thus, DFT calculations were performed to gain more insight into the geometric and electronic properties of the $E$ - and $Z$-C392STP isomers and of the $E / Z$ mixture $(84 / 16)$ (synthesis product). Fig. 2 shows the minimum-energy molecular geometry computed at the B3LYP/6-31+G(d) level for the $E$ - and $Z$-C392STP isomers in THF (the geometries in the other solvents were found to be very similar). The $E$ isomer shows a nearly coplanar structure, suggesting efficient pi conjugation through the molecule, while the $Z$ isomer is twisted out of plane of the carbon-carbon double bond due to steric hindrance. The oscillator strength $(f)$ and the maximum absorption wavelengths of the key-transitions for both isomers in different solvents were calculated (ESI, $\dagger$ Table S1). The energies found for the $E$ isomer are around 7-8 kcal mol ${ }^{-1}$ lower than those of the $Z$ isomer (ESI, $\dagger$ Table S2), independent of the solvent used, as expected, since the bulky groups on the same side of the $Z$ isomer cause repulsive interactions forcing the aromatic ring out-of-plane. These results show that the $E$ isomer is the most stable form and are in accordance with the NMR spectroscopic results, which show the $E$ isomer as the most abundant species. 


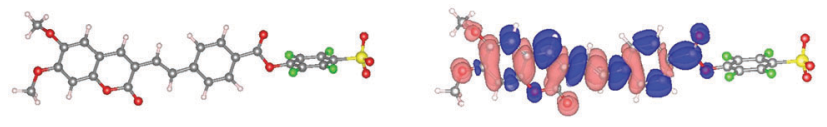

E-C392STP

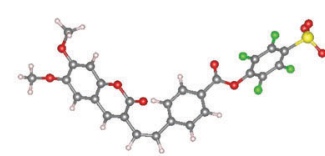

Z-C392STP

Fig. 2 Optimized molecular geometry for $E$ - and Z-C392STP in THF at the B3LYP/6-31+G(d) level (left) where oxygen, carbon, sulfur, fluoride and hydrogens atoms are marked in red, gray, yellow, green and white, respectively. Contour plots of the electron density difference $(\Delta \rho)$ of the lowest energy excitation for the $E$ and $Z$ conformers (right), where blue indicates a positive contribution (increase of electron density) while pink represents a depletion of electron density.

The solvatochromic behaviour of the C392STP isomers and of the $E / Z$ mixture (84/16) was analyzed using Time Dependent Density Functional Theory (TDDFT) methods. The calculations were performed using the Polarizable Continuum Model (PCM) for THF, methanol and water. The simulated absorption spectra, for both the isomers as well as for their mixture, and their comparison with the experimental spectra can be found in the ESI $\dagger$ (Fig. S1). The calculated lower energy transitions in terms of energy and oscillator strength, together with the experimental absorption maxima, are depicted in Table 1.

The lowest-energy $S_{0} \rightarrow S_{1}$ transition can be ascribed to $\pi \rightarrow \pi^{*}$ electron transitions and is mainly of HOMO $\rightarrow$ LUMO character, for both conformers. An illustrative picture of the charge rearrangement that occurs upon the $S_{0} \rightarrow S_{1}$ transitions in THF is given in Fig. 2. The difference between the electronic densities of the states involved in the transitions is depicted, revealing more clearly the regions of the chromophore that lose or gain electrons. The calculated lowest energy transition, $S_{0} \rightarrow S_{1}$, in different solvents (Table 1 ), shows very good agreement with the experimentally measured wavelengths of the coumarin derivative chromophore absorption in non-aqueous solvents (Table 2). However, it is not capable of reproducing the blue shift that occurs in water. This can be attributed to the use of continuum models in which specific interactions between the solute and the solvent need special considerations. Therefore, an explicit solvent cluster model was also used to model the absorption spectra of the isomers in aqueous solutions. In this

Table 1 Calculated absorption data of the lowest energy transition for both isomers in different solvents

\begin{tabular}{|c|c|c|c|c|c|c|}
\hline \multirow[b]{2}{*}{ Solvent } & \multicolumn{2}{|c|}{$E$ isomer } & \multicolumn{2}{|c|}{$Z$ isomer } & \multirow{2}{*}{$\frac{E / Z \text { mixture }}{\lambda^{a}(\mathrm{~nm})}$} & \multirow{2}{*}{$\frac{\text { Experimental }}{\lambda, \mathrm{FWHM}^{b}(\mathrm{~nm})}$} \\
\hline & $\lambda(\mathrm{nm}) J$ & & $\lambda(\mathrm{nm})$ & & & \\
\hline THF & 409 & 1.773 & 384 & 0.870 & 408 & 399,68 \\
\hline Methanol & 410 & 1.753 & 388 & 0.847 & 409 & 396,69 \\
\hline Water & 411 & 1.753 & 389 & 0.824 & 410 & 385,74 \\
\hline Explicit water & 406 & 1.826 & 375 & 0.786 & 405 & \\
\hline
\end{tabular}

${ }^{a}$ The presented values refer to the maxima of the convoluted bands.

${ }^{b}$ FWHM, full width at half-maximum values. model the solvation of both isomers of C392STP was performed with a total of 9 explicit water molecules. The coumarin-water complex was optimized and its absorption spectra were calculated by TDDFT, embedded in a PCM water medium (Table S1, ESI $\dagger$ ). When compared with the continuum model, the explicit water model excitation corresponding to the $S_{0} \rightarrow S_{1}$ transition shifts to higher energies than in non-aqueous solvents (Table 1), as observed experimentally (Table 2), although with a smaller blue shift than in the experiment.

\section{Experimental results}

The absorption and steady-state fluorescence spectra of C392STP were recorded in solvents with different physical properties including different dipolarity/polarizability and hydrogen-bonding ability (Fig. 1C). The maximum absorption and emission wavelengths ( $\lambda_{\mathrm{abs}}$ and $\lambda_{\mathrm{em}}$, respectively) are summarized in Table 2 .

\section{Absorption}

The absorption spectra of C392STP showed a broad absorption band in the region 300-500 $\mathrm{nm}$ (Fig. 1D) with maximum at 394-400 $\mathrm{nm}$ in non-aqueous solvents (Fig. 1D and Table 2). The absence of solvent-polarity dependence of the absorption maximum in non-aqueous solvents might imply that the ground state energy distribution is not affected to a great extent by the polarity of the solvent. This is possibly due to the low polar nature of the dye in the ground state. However, the change of solvent, from non-aqueous to aqueous, causes a slight blue shift of around 11-15 nm $\left(\lambda_{\mathrm{abs}}=385 \mathrm{~nm}\right.$ in water and $\lambda_{\mathrm{abs}}=400 \mathrm{~nm}$ in DMSO). This shift can be attributed to specific solvent-solute interactions, and more specifically to hydrogen-bonding interactions in which the coumarin moieties act as hydrogen acceptors, since the proton-donating capability of water is higher than that of other protic solvents (see SA parameter Table 3).

\section{Emission}

Blue emission was observed in polar solvents for the $E / Z$ isomer mixture. The bright blue fluorescence of C392STP under irradiation with $365-366 \mathrm{~nm}$ ultraviolet light is detectable with the naked eye (Fig. 1C). This could be of great interest for its application in a wide variety of fields. The emission spectra showed only one broad band in the 400-600 $\mathrm{nm}$ region and the maxima are strongly solvent dependent. An increase of the solvent polarity induced a red shift of the wavelength of maximum absorption in both aprotic and protic solvents (from $468 \mathrm{~nm}$ in dioxane to $499 \mathrm{~nm}$ in DMSO). This fact (Table 2) indicates an increase in polarity (dipole moment) upon excitation.

A direct linear correlation was found between $\lambda_{\mathrm{em}}$ or $\Delta \lambda$ and $\varepsilon_{\mathrm{r}}$ for most of the solvents used (Fig. 3A). However, the deviations from linearity indicated that, whereas the relative permittivity is one of the main factors governing the solvent shift it is not the sole one. This was confirmed using the empirical solvent polarity index or normalized transition energy, $E_{\mathrm{T}}^{\mathrm{N}}$, for studying the solvent-solute interactions. ${ }^{34}$ The plot of the Stokes shift of the dye in different solvents against the solvent empirical polarity scale gave linear correlations for two separated solvent classes, protic and aprotic (Fig. 3B). 
Table 2 Solvent effects on the spectral data of C392STP

\begin{tabular}{|c|c|c|c|c|c|c|c|c|c|c|}
\hline \multirow{2}{*}{\multicolumn{2}{|c|}{ Solvent }} & \multirow[b]{2}{*}{$\lambda_{\mathrm{abs}}(\mathrm{nm})$} & \multirow[b]{2}{*}{$\lambda_{\mathrm{em}}(\mathrm{nm})$} & \multirow[b]{2}{*}{$\nu_{\mathrm{abs}}\left(\mathrm{cm}^{-1}\right)$} & \multirow[b]{2}{*}{$\nu_{\mathrm{em}}\left(\mathrm{cm}^{-1}\right)$} & \multicolumn{2}{|c|}{ Stokes shift } & \multirow[b]{2}{*}{$E_{\mathrm{abs}}\left(\mathrm{kcal} \mathrm{mol}^{-1}\right)$} & \multirow[b]{2}{*}{$E_{\text {em }}\left(\mathrm{kcal} \mathrm{mol}^{-1}\right)$} & \multirow[b]{2}{*}{$\Delta E\left(\mathrm{kcal} \mathrm{mol}^{-1}\right)$} \\
\hline & & & & & & $\Delta \lambda(\mathrm{nm})$ & $\Delta \nu(\mathrm{nm})$ & & & \\
\hline 1 & DMSO & 400 & 499 & 25000 & 20040 & 99 & 4960 & 71.48 & 57.30 & 14.18 \\
\hline 3 & Acetone & 396 & 486 & 25253 & 20576 & 90 & 4676 & 72.20 & 58.83 & 13.37 \\
\hline 4 & THF & 399 & 482 & 25063 & 20747 & 83 & 4316 & 71.66 & 59.32 & 12.34 \\
\hline 5 & Ethyl acetate & 396 & 478 & 25253 & 20921 & 82 & 4332 & 72.20 & 59.81 & 12.39 \\
\hline 6 & Dioxane & 394 & 468 & 25381 & 21368 & 74 & 4013 & 72.57 & 61.09 & 11.47 \\
\hline 9 & $40 \%(\mathrm{~A})-60 \%$ (B) & 397 & 499 & 25189 & 20040 & 102 & 5149 & 72.02 & 57.30 & 14.72 \\
\hline 10 & $20 \%(\mathrm{~A})-80 \%(\mathrm{~B})$ & 399 & 493 & 25063 & 20284 & 94 & 4779 & 71.66 & 57.99 & 13.66 \\
\hline 11 & Methanol & 396 & 495 & 25253 & 20202 & 99 & 5051 & 72.20 & 57.76 & 14.44 \\
\hline 12 & Ethanol (B) & 394 & 486 & 25381 & 20576 & 92 & 4805 & 72.57 & 58.83 & 13.74 \\
\hline 13 & Propanol & 395 & 482 & 25316 & 20747 & 87 & 4570 & 72.38 & 59.32 & 13.06 \\
\hline 14 & 1-Butanol & 395 & 484 & 25316 & 20661 & 89 & 4655 & 72.38 & 59.07 & 13.31 \\
\hline
\end{tabular}

Table 3 Physical properties, empirical solvent polarity indexes, and Kamlet-Taft and Catalan parameters of selected solvents

\begin{tabular}{|c|c|c|c|c|c|c|c|c|c|c|c|}
\hline \multirow{2}{*}{\multicolumn{2}{|c|}{ Solvent }} & \multirow[b]{2}{*}{$\varepsilon_{\mathrm{r}}^{a}$} & \multirow[b]{2}{*}{$\eta^{b}$} & \multirow[b]{2}{*}{$E_{\mathrm{T}}^{\mathrm{N} c}$} & \multicolumn{3}{|c|}{ Kamlet-Taft parameters } & \multicolumn{4}{|c|}{ Catalan parameters } \\
\hline & & & & & $\alpha$ & $\beta$ & $\pi^{*}$ & SA & SB & SP & SdP \\
\hline 1 & DMSO & 47.24 & 1.4793 & 0.444 & 0 & 0.76 & 1 & 0.072 & 0.647 & 0.83 & 1 \\
\hline 2 & DMF & 36.71 & 1.4305 & 0.386 & 0 & 0.69 & 0.88 & 0.031 & 0.613 & 0.759 & 0.977 \\
\hline 3 & Acetone & 20.56 & 1.3587 & 0.355 & 0.08 & 0.48 & 0.62 & 0 & 0.475 & 0.651 & 0.907 \\
\hline 4 & THF & 7.58 & 1.4072 & 0.207 & 0 & 0.55 & 0.55 & 0 & 0.591 & 0.714 & 0.634 \\
\hline 5 & Ethyl acetate & 6.02 & 1.3724 & 0.228 & 0 & 0.45 & 0.45 & 0 & 0.542 & 0.656 & 0.603 \\
\hline 6 & Dioxane & 2.21 & 1.4224 & 0.164 & 0 & 0.37 & 0.49 & 0 & 0.444 & 0.737 & 0.312 \\
\hline 7 & PBS $22 \mathrm{mM}$ & 79 & 1.3355 & n.a. & n.a. & n.a. & n.a. & n.a. & n.a. & n.a. & n.a. \\
\hline 8 & Water (A) & 78.36 & 1.333 & 1 & 1.17 & 0.47 & 1.09 & 1.062 & 0.025 & 0.681 & 0.997 \\
\hline 9 & $40 \%$ (A)-60\% (B) & 49.06 & 1.3626 & 0.75 & n.a. & n.a. & n.a. & n.a. & n.a. & n.a. & n.a. \\
\hline 10 & $20 \%(\mathrm{~A})-80 \%(\mathrm{~B})$ & 36.81 & 1.3657 & 0.71 & n.a. & n.a. & n.a. & n.a. & n.a. & n.a. & n.a. \\
\hline 11 & Methanol & 32.66 & 1.3284 & 0.762 & 0.98 & 0.66 & 0.6 & 0.605 & 0.545 & 0.608 & 0.904 \\
\hline 12 & Ethanol (B) & 24.55 & 1.3614 & 0.654 & 0.86 & 0.75 & 0.54 & 0.4 & 0.658 & 0.633 & 0.783 \\
\hline 13 & Propanol & 20.45 & 1.3856 & 0.617 & 0.84 & 0.9 & 0.52 & 0.367 & 0.727 & 0.658 & 0.748 \\
\hline 14 & 1-Butanol & 17.51 & 1.3993 & 0.586 & 0.84 & 0.84 & 0.47 & 0.341 & 0.809 & 0.674 & 0.655 \\
\hline
\end{tabular}

n.a.: data not available in the literature or not found by us. ${ }^{a}$ Dielectric constant. ${ }^{b}$ Refractive index. ${ }^{c}$ Molecular-microscopic solvent polarity parameter. The values of these parameters were taken from ref. 34 .
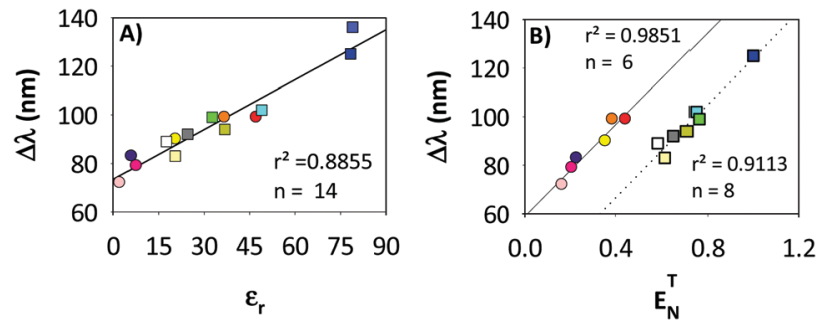

Fig. 3 Stokes shift dependence on (A) the permittivity of the medium $\left(\varepsilon_{r}\right)$ and $(B)$ the normalized transition energy $\left(E_{N}^{\top}\right)$.

This behaviour has also been found by other authors investigating the solvatochromism of coumarins as well as other compounds. ${ }^{25}$ It can be explained considering that the $E_{\mathrm{N}}^{\mathrm{T}}$ parameter represents only the dipolarity/polarizability and acidity contribution of the solvent but not the basicity contribution of the solvent or specific solvent-solute interactions. ${ }^{25,34}$ This revealed that, upon excitation whereas in aprotic solvents only non-specific solvent-solute interactions occur, in protic solvents the contribution of the specific solvent-solute interactions (hydrogen bonding) is considerable. Thus, when solubilized in protic solvents, the hydrogen bond formation stabilizes the excited state of C392STP decreasing its energy. This is in accordance with the red shift of the emission spectrum found in aqueous solutions (see Table 2). Also, the strong bathochromic shift observed in the emission spectra with solvent polarity while the absorption spectra remain unchanged indicates a $\pi-\pi^{*}$ transition $\left(\pi^{*}-\pi\right.$ fluorescence is more sensitive to hydrogen bonding than $\pi-\pi^{*}$ absorption). ${ }^{1}$

\section{Solvent effects on the Stokes shift and emission spectra of C392STP}

As shown in Fig. 1, in all the solvents investigated the spectral overlap between the absorption and fluorescence of the novel coumarin dye was small. Large Stokes shifts (separation between the absorption and emission maxima measured in $\mathrm{nm}$ or $\mathrm{cm}^{-1}$, $\Delta \lambda$ and $\Delta \nu$, respectively) were obtained for this compound (Table 2). They are lower for dioxane $\left(\Delta \lambda_{\text {dioxane }}=74 \mathrm{~nm}\right)$ than for PBS $\left(\Delta \lambda_{\mathrm{PBS}}=136 \mathrm{~nm}\right)$. The Stokes shift obtained for C392STP in PBS is higher than those of common fluorophores with different skeletons such as fluorescein, rhodamine, cyanine, 
Table 4 Photophysical properties of several coumarin dyes with proven application as fluorescent dyes in various fields

\begin{tabular}{|c|c|c|c|c|c|c|c|}
\hline Fluorescent dye & $\lambda_{\mathrm{abs}} / \lambda_{\mathrm{em}}(\mathrm{nm})$ & $\Delta \lambda(\mathrm{nm})$ & $\varepsilon\left(10^{4} \mathrm{M}^{-1} \mathrm{~cm}^{-1}\right)$ & $\Phi$ & Brightness $\left(10^{4} \mathrm{M}^{-1} \mathrm{~cm}^{-1}\right)$ & Solvent & Ref. \\
\hline Pacific Orange & $390 / 540$ & 150 & n.a. & n.a. & n.a. & $\mathrm{H}_{2} \mathrm{O}$ & 12 \\
\hline BD Horizon $^{\mathrm{TM}}$ V500 & $415 / 500$ & 85 & n.a. & n.a. & n.a. & $\mathrm{H}_{2} \mathrm{O}$ & 12 \\
\hline Alexa488 & $495 / 519$ & 24 & 7.1 & 0.92 & 6.53 & PBS & 35 \\
\hline Alexa 594 & $590 / 617$ & 27 & 9.0 & 0.66 & 5.94 & PBS & 35 \\
\hline Atto 390 & $390 / 479$ & 89 & 2.4 & 0.90 & 0.02 & $\mathrm{H}_{2} \mathrm{O}$ & 35 \\
\hline Atto 655 & $663 / 684$ & 21 & 12.5 & 0.30 & 3.75 & PBS & 35 \\
\hline Atto 700 & 700/719 & 19 & 12.0 & 0.25 & 3.00 & PBS & 35 \\
\hline DY-344IN & $342 / 455$ & 113 & 2.7 & n.a. & n.a. & EtOH & 12 \\
\hline DY-350XL & $349 / 610$ & 261 & 1.6 & n.a. & n.a. & EtOH & 12 \\
\hline DY-360XL & $362 / 459$ & 97 & 2.5 & n.a. & n.a. & PBS & 12 \\
\hline DY-370XL & $368 / 473$ & 105 & 1.3 & n.a. & n.a. & PBS & 12 \\
\hline DY-395XL & $396 / 572$ & 176 & 2.0 & n.a. & n.a. & EtOH & 12 \\
\hline CR1E & $596 / 652$ & 56 & 7.2 & 0.01 & 0.07 & $\mathrm{H}_{2} \mathrm{O}$ & 3 \\
\hline CR2E & $602 / 666$ & 64 & 7.6 & $<0.01$ & n.a. & $\mathrm{H}_{2} \mathrm{O}$ & 3 \\
\hline CR3E & $616 / 687$ & 71 & 6.6 & $<0.01$ & n.a. & $\mathrm{H}_{2} \mathrm{O}$ & 3 \\
\hline c1 & $367 / 451$ & 84 & n.a. & n.a. & n.a. & EtOH & 27 \\
\hline c102 & $382 / 473$ & 90 & n.a. & n.a. & n.a. & EtOH & 27 \\
\hline c120 & $350 / 433$ & 83 & n.a. & n.a. & n.a. & EtOH & 27 \\
\hline c151 & $376 / 490$ & 114 & n.a. & n.a. & n.a. & EtOH & 27 \\
\hline c152 & $390 / 519$ & 128 & n.a. & n.a. & n.a. & EtOH & 27 \\
\hline c153 & $422 / 532$ & 110 & n.a. & n.a. & n.a. & EtOH & 27 \\
\hline FR521 & $520 / 700$ & 180 & 3.5 & 0.01 & 0.02 & PBS & 9 \\
\hline
\end{tabular}

n.a.: data not available in the literature or not found by us.

nile red and BODIPY dyes $\left(\Delta \lambda_{\mathrm{PBS}} \leq 70 \mathrm{~nm}\right) .{ }^{11,12}$ Notwithstanding, they are higher (Table 2) than those of several commercially available coumarin dyes (Table 4) including AlexaFluor ${ }^{\mathrm{TM}} 488$ $\left(\lambda_{\mathrm{abs}}=494 \mathrm{~nm}, \lambda_{\mathrm{em}} 519 \mathrm{~nm}\right.$ and $\left.\Delta \lambda_{\mathrm{PBS}}=25 \mathrm{~nm}\right)$ - a commonly used fluorescent dye also commercialized in the TFP ester form for labeling proteins and oligonucleotides. ${ }^{35}$ It is particularly relevant that the Stokes shifts found for the C392STP ester are similar, in terms of magnitude, to those exhibited by some commercially-available coumarins with large Stokes shifts: 'Mega Stokes' dyes from Dyomics $\left(\lambda_{\mathrm{abs}}=500-520 \mathrm{~nm}, \lambda_{\mathrm{em}}=\right.$ 590-670 $\mathrm{nm}$ and $\Delta \lambda=90-150 \mathrm{~nm}$ in ethanol) or AlexaFluor ${ }^{\mathrm{TM}} 430$ $\left(\lambda_{\text {abs }}=434 \mathrm{~nm}, \lambda_{\mathrm{em}} 539 \mathrm{~nm}\right.$ and $\left.\Delta \lambda_{\mathrm{PBS}}=105 \mathrm{~nm}\right) \cdot{ }^{6,12}$ A great Stokes shift, such as that shown by C392STP, is beneficial for practical application since it reduces self-quenching, boosts the signal-to-noise ratio in bioimaging applications and allows multicolor experiments to be performed reducing the number of detection channels, avoiding spectral overlap artifacts (bleed-through or crosstalk) and simplifying the imaging scheme. ${ }^{2,6,11,12}$ Thus, similar to other fluorescent dyes with large Stokes shifts, C392STP could initially be successfully applied as a sensor, molecular probe and light-emitting marker in chemistry, life sciences and optical microscopy.

While studying the influence of the polarity of the solvent on the Stokes shift (Fig. 3A) it was found that C392STP is more susceptible to solvent polarity than other commercial coumarins (with a slope $(m)$ of $m=0.6833\left(r^{2}=0.9322\right)$ AMCA: $m=0.49, r^{2}=0.73$; coumarin 1: $m=0.62, r^{2}=0.71$; coumarin $\left.6: m=0.39, r^{2}=0.2\right) .{ }^{32}$ The strong fluorescence solvatochromism displayed by C392STP points to the possibility of using it as a polarity sensor.

The Stokes shift values of C392STP increased with solvent polarity for both protic and aprotic solvents $25 \mathrm{~nm}$ from dioxane to DMSO and $47 \mathrm{~nm}$ from 1-butanol to PBS). This fact also supports the increase in the dipole moment upon excitation. ${ }^{13,34,36}$ By comparing the Stokes shifts exhibited by C392STP in aprotic and protic solvents with similar dielectric constants (acetone and DMF with propanol and ethanol/water (80/20), respectively) only slight differences were found (3-5 nm larger in aprotic solvents (Tables 2 and 3) with the regression lines $\left(\Delta \lambda / E_{\mathrm{N}}^{\mathrm{T}}\right)$ obtained for both types of solvents being almost parallel, Fig. 3B). The highest Stokes shifts were found in water and PBS. This could be due, again, to the higher protondonating capability of water than of other protic solvents. ${ }^{18}$

Solvent effects on the Stokes shift of dye molecules $(\Delta \nu)$ may arise from physical intermolecular solute-solvent interactions (dipolar interactions) or chemical processes (such as electron or proton transfer, complexation or isomerization). ${ }^{34,36}$ Thus, for gaining insight into the solvent effects on the spectral shifts of C392STP six different empirical solvatochromic models were used: Lippert-Mataga's (LM), Bakhshiev's (B), Kawski-ChammaViallet's (KCV), Ravi's (R), Taft-Kamlet's (TK) and two Catalan's models (C3P and C4P). All these models have already demonstrated their potential for understanding coumarin-solvent interactions and some of them have also been used for calculating the variation of the transition dipole moment upon excitation..$^{2,24,26,27}$ 
The results of the statistical treatment of the data obtained for polar protic and aprotic solvents using the models based on the reaction field theories - LM, B and KCV - and the corresponding plots are summarized in the ESI $\dagger$ (Table S3). No linear correlations were found between the Stokes shifts $(\Delta \nu)$ and the solvent orientation polarity functions, $f_{x}(\varepsilon, n)$ considering all the solvents investigated. The protic and the aprotic solvents are clearly divided into two domains. This behavior has been previously reported in the case of polarity probes like PRODAN and its analogues. ${ }^{37,38}$ This has been attributed to the fact that the models used (LM, B and KCV) do not consider molecular aspects of solvation and chemical interactions and should indicate the existence of a specific solvent effect, most probably intermolecular $\mathrm{H}$-bonding between the solute and the solvent molecules. $^{34,36,37,39}$

When specific interactions between solute and solvent molecules can contribute to the Stokes shifts, models such as Ravi's model, which applies the empirical solvent polarity index, $E_{\mathrm{N}}^{\mathrm{T}}$ (considering dipolarity/polarizability and acidity), are used to investigate the solvent-solute interaction effects on the Stokes shift. Good linear correlations were found for two separated solvent classes, polar protic (excluding PBS $22 \mathrm{mM}$ ) and aprotic, (i) indicating that this model allows accurate modeling of the Stokes shift variation of C392STP in both protic and aprotic solvents (Table 5); and (ii) suggesting the presence of an additional interaction between C392STP and polar protic solvents which is ignored in the reaction field approaches. ${ }^{26}$ The results indicate that the interaction with aprotic (polar but non-hydrogen bonding) solvents depends on dipole-dipole forces, while with protic solvents, additional specific solvent-solute interactions exist. H-bonding notably contributes to the change in the spectra observed for C392STP in these solvents. ${ }^{25}$

Multiparameter models that independently quantify the influence of both specific and non-specific solvent-solute interactions (KT, C3P and C4P) generally work better for investigating the solvent effects on the absorption and emission spectra, as well as on the Stokes shifts of coumarin dyes, than the reaction field model and Ravi's method. ${ }^{2,27}$ Thus, they were used to investigate the effect of solvent dipolarity/polarizability and hydrogen bonding on the emission maxima $\left(\nu_{\mathrm{em}}\right)$ and the Stokes shifts $\left(\Delta \nu=\nu_{\mathrm{abs}}-\nu_{\mathrm{em}}\right)$, all in $\mathrm{cm}^{-1}$. The multiple linear regressions can be described by the following equations:

$$
\begin{gathered}
\nu=\nu_{0}+a \alpha+b \beta+c \pi^{*} \quad(\mathrm{KT}) \\
\nu=\nu_{0}+a \mathrm{SA}+b \mathrm{SB}+c \mathrm{SPP} \quad(\mathrm{C} 3 \mathrm{P}) \\
\nu=\nu_{0}+a \mathrm{SA}+b \mathrm{SB}+c \mathrm{SP}+d \mathrm{SdP} \quad(\mathrm{C} 4 \mathrm{P})
\end{gathered}
$$

where $\nu_{0}$ represents the physicochemical property of interest in the absence of solvent (i.e. in the gas phase). The KamletTaft model parameters $\alpha, \beta$ and $\pi^{*}$ denote the hydrogen bond donor (HBD) ability, hydrogen bond acceptor (HBA) ability and polarity (dipolarity and polarizability jointly) of the solvents, respectively. The Catalan model parameters SA and SB characterize specific interaction effects, solvent acidity and basicity, respectively, and the polarity SPP, unspecific solvent effects, which can be separated into two parameters: solvent polarizability (SP) and dipolarity (SdP). $a-d$ are the regression coefficients and their magnitude and sign describe the sensitivity of

\begin{tabular}{|c|c|c|c|c|c|c|}
\hline \multicolumn{7}{|c|}{ Ravi $\left(\nu=\nu_{0}+11307.6 a E_{\mathrm{N}}^{\mathrm{T}}+b\right)$} \\
\hline$\nu_{\mathrm{em}}\left(\mathrm{cm}^{-1}\right)$ & Protic & $22757(214)$ & $-3435(292)$ & 0.9652 & 0.9583 & 7 \\
\hline$\Delta \nu\left(\mathrm{cm}^{-1}\right)$ & Protic & $2788(284)$ & $1195(187)$ & 0.9105 & 0.8882 & 7 \\
\hline$\Delta \nu\left(\mathrm{cm}^{-1}\right)$ & Aprotic & $1488(449)$ & 4967 (609) & 0.9301 & 0.9161 & - \\
\hline
\end{tabular}

Table 5 Results of the statistical treatment of the Ravi, Taft-Kamlet and Catalan correlations considering two parameters, $P$ (the emission frequencies and Stokes shift), in the solvents selected: slopes $(m)$, intercepts $(p)$ and correlation coefficients $\left(R^{2}\right.$ and $\left.\operatorname{adj} R^{2}\right)$

\begin{tabular}{|c|c|c|c|c|c|c|c|c|c|c|c|}
\hline$\nu$ & $\nu_{\mathrm{o}}$ & $a$ & $b$ & $c$ & $\operatorname{adj} R^{2}$ & $n^{a}$ & $F^{b}$ & $P_{\mathrm{a}}^{c}(\%)$ & $P_{\mathrm{b}}^{c}(\%)$ & $P_{\mathrm{c}}^{c}(\%)$ & S.E. \\
\hline$\Delta \nu\left(\mathrm{cm}^{-1}\right)$ & $2994(220)$ & $726(143)$ & 0 & $2314(313)$ & 0.8938 & 11 & 43 & 24 & 0 & 76 & $7,9,10$ \\
\hline \multicolumn{12}{|c|}{ Catalan $3 \mathrm{P}\left(\nu=\nu_{0}+a \mathrm{SA}+c \mathrm{SPP}\right)$} \\
\hline$\nu$ & $\nu_{0}$ & $a$ & $b$ & $c$ & $\operatorname{adj} R^{2}$ & $n^{a}$ & $F^{b}$ & $P_{\mathrm{a}}^{c}(\%)$ & $P_{\mathrm{b}}^{c}(\%)$ & $P_{\mathrm{c}}^{c}(\%)$ & S.E. \\
\hline$\nu_{\mathrm{em}}\left(\mathrm{cm}^{-1}\right)$ & $24632(425)$ & $-741(122)$ & 0 & $-4556(499)$ & 0.9437 & 11 & 85 & 14 & 0 & 86 & $7,9,10$ \\
\hline
\end{tabular}

Taft-Kamlet $\left(\nu=\nu_{0}+a \alpha+c \pi^{*}\right)$

\begin{tabular}{|c|c|c|c|c|c|c|c|c|c|c|c|c|c|}
\hline$\nu$ & $\nu_{0}$ & $a$ & $b$ & $c$ & $d$ & $\operatorname{adj} R^{2}$ & $n^{a}$ & $F^{b}$ & $P_{\mathrm{a}}^{c}(\%)$ & $P_{\mathrm{b}}^{c}(\%)$ & $P_{\mathrm{c}}^{c}(\%)$ & $P_{\mathrm{d}}^{c}(\%)$ & S.E. \\
\hline$\nu_{\mathrm{em}}\left(\mathrm{cm}^{-1}\right)$ & $23506(513)$ & $-878(154)$ & 0 & $-2310(750)$ & $-1532(224)$ & 0.9371 & 11 & 51 & -19 & 0 & 49 & 32 & 7,9 \\
\hline$\Delta \nu\left(\mathrm{cm}^{-1}\right)$ & 1588 (965) & $1541(289)$ & 0 & $2680(1411)$ & $1267(422)$ & 0.8667 & 11 & 23 & 28 & 0 & 49 & 23 & $7,9,10$ \\
\hline
\end{tabular}

Catalan $4 \mathrm{P}\left(\nu=\nu_{0}+a \mathrm{SA}+c \mathrm{SP}+d \mathrm{SdP}\right)$

${ }^{a}$ Number of solvents included in the correlation. ${ }^{b}$ Fisher's tests. ${ }^{c}$ Percentage contribution of solvatochromic parameters. 
the property $\nu$ to the different types of solvent-solute interactions. For obtaining reliable results for solutes such as C392STP, capable of accepting hydrogen bonds from a protic solvent but not capable of donating hydrogen bonds, physically invalid terms in the best-fit equations derived from the KT, C3P and C4P models were avoided forcing the values of $b$ to null. ${ }^{2}$ The equations used, the solvatochromic parameters thus obtained and their percentage contribution as well as the solvents excluded are summarized in Table 5. Only the physically valid best-fit, using the $F$ and $\operatorname{adj} R^{2}$ values as criteria for its determination, are shown.

The three models gave satisfactory results and the regression coefficient sets obtained ( $a, c$ and $d$ ) and their percentage contributions $\left(P_{a}, P_{c}\right.$ and $\left.P_{d}\right)$ are in good agreement between them. Thus, the Kamlet-Taft and Catalan approaches demonstrated excellent statistical performance in describing the solvatochromism of C392STP spectra due to specific and nonspecific interactions with the solvents. Furthermore, the best-fit coefficients of these models allow deeper insight to be gained into the nature of the solvent-coumarin interactions. The values of $a, c$ and $d$ are relatively large indicating that the hydrogen bond aceptor (HBA) ability and the polarity of the solvents are the major effects on the solvatochromic change. ${ }^{2,25,38}$ The negative sign of these coefficients for $\nu_{\mathrm{em}}$ and positive signs for $\Delta \nu$ regressions indicate that an increment of the solvent hydrogen-bond acidity, the solvent polarizability or the solvent dipolarity produces a bathochromic shift in the emission spectra and greater stabilization of the excited state compared to the ground state revealing a larger increase in the dipole moment of the excited state than of the ground state. ${ }^{18,40}$

Considering the percentage contribution of the solvatochromic parameters obtained with the KT and C3P models it can be concluded that the polarity of the solvent is the dominant effect (with 75 and $86 \%$ contribution for the changes in $\nu_{\mathrm{em}}$ and $\Delta \nu$, respectively). However, H-bonding also plays a key role in the red shifting of the C392STP emission spectra (25\% contribution). The Catalán 4P model (C4P) offers insights into the roles of solvent polarizability in C392STP spectral shifts. The results obtained indicated that solvent polarizability is the most critical factor (representing a contribution of $49 \%$ ). Also, the transition energy of C392STP in vacuo can be predicted from the fits for the three multiparameter models since the values obtained are in reasonable agreement between them (Table 5).

\section{Molar absorption coefficient $(\varepsilon)$, quantum yield $(\Phi)$ and brightness $(\varepsilon \times \Phi)$}

High molar extinction coefficients and quantum yields are preferred in general for fluorescent dyes, as their product corresponds to the fluorescence brightness. ${ }^{2}$ The molar extinction coefficients of C392STP in PBS, water, acetone and DMSO were determined at the wavelengths of maximum absorption. Their values were found to be moderate in all these solvents $\left(\varepsilon_{385}=1.03 \times 10^{4} \mathrm{M}^{-1} \mathrm{~cm}^{-1}, \varepsilon_{388}=1.85 \times 10^{4} \mathrm{M}^{-1} \mathrm{~cm}^{-1}, \varepsilon_{396}=\right.$ $2.0 \times 10^{4} \mathrm{M}^{-1} \mathrm{~cm}^{-1}, \varepsilon_{400}=1.98 \times 10^{4} \mathrm{M}^{-1} \mathrm{~cm}^{-1}$, respectively) when compared with the values obtained for other compounds and coumarins (Table 4).
The quantum yield was calculated exclusively in PBS. Two quantum yield standards were used as reference: Quinine Sulfate (QS) in $0.5 \mathrm{M} \mathrm{H}_{2} \mathrm{SO}_{4}\left(\Phi_{\mathrm{R}}=0.546\right)$ and 9,10-diphenylanthracene (DPA) in cyclohexane $\left(\Phi_{\mathrm{R}}=0.955\right)$. The quantum yield of C392STP in PBS was determined to be 0.115 (mean of the values obtained using both references, 0.11 with QS and 0.12 with DPA, respectively). This value is relatively low, lower than those obtained for commercially available coumarins but much higher than those of various synthesized coumarins described previously (Table 4).

The values of the molar extinction coefficient and quantum yield, in agreement with the results obtained in silico and with the lack of shift of the absorption spectrum and the red shift of the emission spectrum with increasing solvent polarity, revealed a $\pi-\pi^{*} S_{0}-S_{1}$ transition.

Low brightness has been referred to as one of the main drawbacks of many fluorescent dyes with large Stokes shifts. ${ }^{12}$ However, that is not the case here, as the brightness of C392STP has been found to be moderate $\left(\varepsilon \times \Phi=1.18 \times 10^{3} \mathrm{M}^{-1} \mathrm{~cm}^{-1}\right)$, similar to those obtained for other synthesized coumarins with very large Stokes shifts, upper than $100 \mathrm{~nm}$, suitable for bioconjugation and STED microscopy. ${ }^{6}$ The brightness of this novel coumarin is higher than those of other synthesized coumarins successfully used as chemosensors or for biolabeling and even higher than that of a commercially available dye, Atto 390, with application in labeling of biomolecules (Table 4).

\section{Behaviour of C392STP in aqueous solution}

Influence of pH. Rhodamine and fluorescein dyes as well as some coumarins, commercially available (including Alexa dyes), have maintained their popularity because of their greater resistance to photodegradation and $\mathrm{pH}$ insensitivity. ${ }^{41}$ Variation of the $\mathrm{pH}$ can modify the absorption and emission properties of the fluorescent dyes and determine their applicability. Thus, the influence of $\mathrm{pH}$ on the properties of the dyes must be carefully examined before their use.

The $\mathrm{pH}$ dependence of the absorbance and fluorescence intensity of C392STP was investigated in the interval 2-9.2 (Fig. 4). This fluorophore was shown to be $\mathrm{pH}$-insensitive within the broad range of $\mathrm{pH}$ studied (its absorption and emission spectra remain constant over the range studied, Fig. 4). This indicates that C392STP, a mixture of $E$ and $Z$ isomers, is a suitable fluorescence imaging reagent under physiological conditions. Furthermore, this characteristic also points out the suitability of C392STP for biolabeling as most of the labelling reactions are carried out in the $\mathrm{pH}$ range $7-9$.

Photostability. It is well-known that the suitability of some fluorophores as fluorescent dyes in different fields and techniques is strongly dependent on their photostability. Thus, the photostability of C392STP was investigated. It was done by monitoring the absorption and emission spectra of an aqueous solution of the dye directly irradiated with a mercury lamp $(365 \mathrm{~nm})$. Aliquots of the solution were analyzed at different time intervals. Fig. 5 shows the changes in the absorption and fluorescence intensity observed as a function of irradiation time with $365 \mathrm{~nm}$ light (power: $50 \mathrm{~W}$ ). The exposure to UV light 

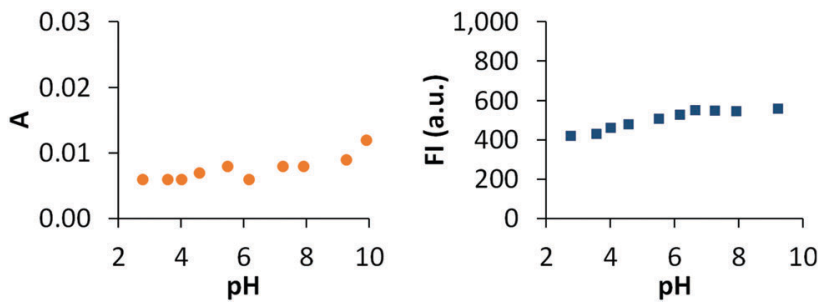

Fig. 4 Absorption and emission intensities recorded at $400 \mathrm{~nm}$ and $500 \mathrm{~nm}$, respectively, versus the $\mathrm{pH}$ of the solution.

produced a decay of the optical density and of the fluorescence intensity at 300-450 $\mathrm{nm}$ and at 450-600 $\mathrm{nm}$, respectively.

The variations in the spectra observed over time revealed that (i) both isomers undergo photobleaching under UV illumination and; (ii) the maxima of emission of both isomers are different and allow their separated detection. No detectable shift of the wavelength of maximum absorption was observed over time. After 35 min of continuous irradiation the absorbance was reduced

\section{In water}
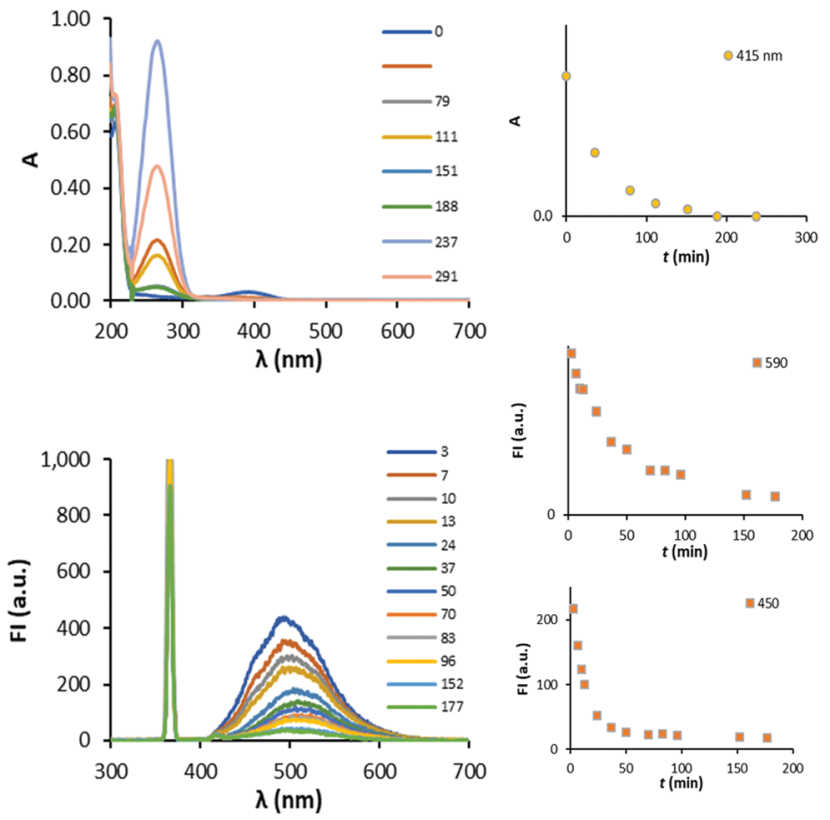

\section{In DMSO}

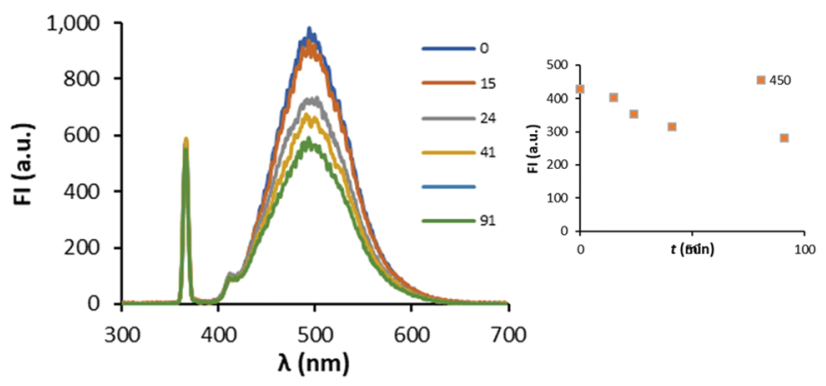

Fig. 5 Investigation of the photostability of C392STP by continuous illumination with a UV lamp (365 nm) in water and in DMSO. to $42 \%$ and completely disappeared after $3 \mathrm{~h}$ (188 min). However, a bathochromic shift in the maximum of emission indicated that $E$ - and Z-C392STP isomers undergo photobleaching at different rates. The profiles of the decay of fluorescence intensity over time also support this finding (Fig. 5). The isomer that emits at higher wavelengths is more unstable than that emitting at lower wavelengths. After $35 \mathrm{~min}$ the fluorescence intensity decayed to $7.9 \%$ at $450 \mathrm{~nm}$ and to $38 \%$ at $590 \mathrm{~nm}$. The isomers were completely photobleached after 1 and $3 \mathrm{~h}$, respectively.

This is interesting from the point of view of detection, because for techniques that require short illumination times, detection at shorter wavelengths around $450 \mathrm{~nm}$ is preferred (the fluorescence intensities are initially four-times higher than that at $590 \mathrm{~nm}$ ). However, for analysis that requires longer periods of exposure to UV light, detection of the fluorescence intensity at longer wavelengths is recommended.

The behavior observed in DMSO was completely different. In DMSO the wavelengths of maximum emission of both isomers are similar. Whereas one of the isomers suffered photobleaching, the other remained stable after $40 \mathrm{~min}$ (Fig. 5). This could be interesting for applications that do not require aqueous media.

\section{Experimental}

\section{Materials and methods}

The solvents used were of spectroscopic grade (Sigma and Merck). A $22 \mathrm{mM}$ Phosphate Buffered Saline solution (PBS) was prepared in milliQ water $\left(\mathrm{Na}_{2} \mathrm{HPO}_{4} 1.18 \mathrm{mM}, \mathrm{KH}_{2} \mathrm{PO}_{4} 0.22 \mathrm{mM}, \mathrm{NaCl} 20.20 \mathrm{mM}\right.$, $\mathrm{KCl} 0.40 \mathrm{mM}, \mathrm{pH}$ 7.2). The quantum yield standards selected, quinine sulfate and 9,10-diphenylanthracene, were purchased from Sigma. The range of solvents was limited by the low solubility of the polar coumarin in low and non-polar liquids. The physical properties and polarity parameters of all solvents used in the study are listed in Table 3: relative permittivity, $\varepsilon_{\mathrm{r}}$, refractive index, $\eta$, the $E_{\mathrm{N}}^{\mathrm{T}} 34$ and the corresponding Kamlet-Taft ${ }^{42}$ and Catalan ${ }^{43}$ solvent parameters. They are ordered according to their decreasing permittivity among two different sets: protic and aprotic.

\section{Equipment}

Absorption and emission spectra were recorded using a Hitachi model U-3010 spectrophotometer and a Hitachi F-2000 spectrofluorometer, respectively. For recording the emission spectra all the samples were excited at $366 \mathrm{~nm}$. All spectra were recorded using $1 \mathrm{~cm}$ pathlength synthetic quartz cuvettes at room temperature keeping the dye concentration very low to avoid self-absorption. A handheld, pH $1000 \mathrm{H}$, pHenomenal ${ }^{\circledR} \mathrm{pH} / \mathrm{mV} /{ }^{\circ} \mathrm{C}$ meter was used for $\mathrm{pH}$ measurements.

Determination of the molar absorption coefficient $(\varepsilon)$, quantum yield $(\Phi)$ and brightness $(\varepsilon \times \Phi)$

The molar absorption coefficients were determined from eight separately weighed solutions with concentrations in the range $0.5-5 \mu \mathrm{M}$. The concentrations were chosen in such a way that the absorbances of the sample solutions did not exceed 0.2 (a.u.). The relative quantum yield of coumarin in PBS, $\Phi_{\mathrm{C}}$, was determined 
using the relative method of Williams et $a .^{44}$ and was calculated through the following equations:

$$
\begin{gathered}
\Phi_{\mathrm{C}}=\Phi_{\mathrm{R}}\left(\frac{\Delta_{\mathrm{FI}_{\mathrm{C}}} / \Delta_{\mathrm{A}_{\mathrm{C}}}}{\Delta_{\mathrm{FI}_{\mathrm{R}}} / \Delta_{\mathrm{A}_{\mathrm{R}}}}\right)\left(\frac{\eta_{\mathrm{C}}^{2}}{\eta_{\mathrm{R}}^{2}}\right) \\
\Phi_{\mathrm{C}}=\Phi_{\mathrm{R}}\left(\frac{m_{\mathrm{C}}}{m_{\mathrm{R}}}\right)\left(\frac{\eta_{\mathrm{C}}^{2}}{\eta_{\mathrm{R}}^{2}}\right)
\end{gathered}
$$

where $\Delta_{\mathrm{FI}}$ is the integrated area under the corrected fluorescence spectrum, $\Delta_{\mathrm{A}}$ is the optical density at the excitation wavelength $\left(\lambda_{\mathrm{ex}}=366 \mathrm{~nm}\right), m$ is the slope of the linear regression $\Delta_{\mathrm{FI}} / \Delta_{\mathrm{A}}$, and $\eta$ is the refractive index of the solution. The subscripts $\mathrm{C}$ and $\mathrm{R}$ refer to the coumarin and reference solutions, respectively. Quinine sulfate in $0.5 \mathrm{M} \mathrm{H}_{2} \mathrm{SO}_{4}\left(\Phi_{\mathrm{R}}=0.546\right)$ and 9,10-diphenylanthracene in cyclohexane $\left(\Phi_{\mathrm{R}}=0.955\right)$ were used as quantum yield reference standards.

For calculating the relative quantum yield, the emission spectra of eight separately weighed solutions with absorbances of less than 0.1 at the first absorption maximum were recorded.

\section{Computational details}

Density functional theory and time dependent density functional calculations were performed with the Gaussian 16 software package. ${ }^{45}$ Geometry optimizations were performed with the B3LYP functional ${ }^{46,47}$ in the different solvents. The standard $6-31+\mathrm{G}(\mathrm{d})$ basis set was employed as it represents a good compromise between accuracy and computational efficiency. Frequency calculations on the optimized geometries presented no imaginary frequencies. For the TD-DFT calculations the hybrid PBE0 functional ${ }^{48}$ together with the much larger $6-311+g(2 d, p)$ basis set was employed. Solvent effects were modelled by means of the implicit polarized continuum (PCM) model. ${ }^{49,50}$

Previous studies have shown that this scheme (B3LYP for geometry optimization, followed by TD-DFT with the PBE0 functional and PCM model to take the solvent into account) gives reliable results for many organic dyes ${ }^{51-55}$ including coumarins. ${ }^{56-58}$

\section{Investigation of the solvent effects and calculation of the excited state dipole moment}

Various equations were used for investigating the solvent effects on the photophysical properties of C392STP. Three were based on quantum mechanical calculations: Lippert-Mataga, the Bakhsiev equation and Kawski-Chamma-Viallet (ESI, $\uparrow$ Table S3).

Also, a relationship based on the empirical polarity scale of Reichardt, $E_{\mathrm{N}}^{\mathrm{T}}$, was used and three Linear Solvation Energy Relationships (LSER) were used for evaluating the influence of the solvent dipolarity/polarizability and hydrogen bonding on the absorption spectra: Kamlet-Taft, Catalan 3P and Catalan $4 \mathrm{P}$ (Table 5).

\section{Conclusions}

In this work interesting physicochemical characteristics of the Coumarin392STP ester were discovered. Also, the behavior of this dye in different solvents, particularly in aqueous solutions, was investigated in detail. The properties observed, similar to those of commercial dyes or even better (i.e. the high Stokes shift), point to the possibility of C392STP, a novel low-cost dye with capacity to react with primary amine groups, becoming a fluorescent dye with a wide range of applications in bioimaging and biolabeling. Some of the advantages of this fluorescent dye are facile preparation with high yield, large Stokes shift, $\mathrm{pH}$-independence of absorbance and emission and excellent photo-stability, which are beneficial for biological fluorescence imaging.

\section{Conflicts of interest}

There are no conflicts to declare.

\section{Acknowledgements}

This work was co-financed by FCT - Fundação para a Ciência e a Tecnologia through the project "MICROTECH-ARTMicroorganisms Thriving on and Endamaging Cultural Heritage -an Analytical Rapid Tool-" (PTDC/BBB-IMG/0046/2014) and by the European Union, European Regional Development Fund ALENTEJO 2020 through the project "MEDUSAMicrorganisms Monitoring and Mitigation-Developing and Unlocking novel Sustainable Approaches-"(ALT20-03-0145FEDER-000015). Marina González-Pérez and Sérgio Martins acknowledge FCT for the economic support through the postdoctoral and doctoral grants SFRH/BPD/100754/2014 and SFRH/BD/128807/2017, respectively. The authors also acknowledge Professor H. Heinz and the referees of the manuscript for their valuable comments and suggestions.

\section{References}

1 A. E. Lanterna, M. González-Béjar, M. Frenette and J. C. Scaiano, Photochem. Photobiol. Sci., 2017, 16, 1284-1289.

2 X. Liu, J. M. Cole and K. S. Low, J. Phys. Chem. C, 2013, 117, 14731-14741.

3 H. Li, L. Cai and Z. Chen, Adv. Chem. Sens., 2012, 121-150.

4 A. Gandioso, R. Bresolí-Obach, A. Nin-Hill, M. Bosch, M. Palau, A. Galindo, S. Contreras, A. Rovira, C. Rovira, S. Nonell and V. Marchán, J. Org. Chem., 2018, 83, 1185-1195.

5 G. Wenska, M. Insińska and B. Skalski, Pol. J. Chem., 2000, 74, 659-671.

6 H. Schill, S. Nizamov, F. Bottanelli, J. Bierwagen, V. N. Belov and S. W. Hell, Chem. - Eur. J., 2013, 19, 16556-16565.

7 J. Chen, W. Liu, B. Zhou, G. Niu, H. Zhang, J. Wu, Y. Wang, W. Ju and P. Wang, J. Org. Chem., 2013, 78, 6121-6130.

8 R. P. Bhusal, P. Yun Cho, S. A. Kim, H. Park and H. S. Kim, Bull. Korean Chem. Soc., 2011, 32, 1461-1462. 
9 B. Wetzl, M. Gruber, B. Oswald, A. Dürkop, B. Weidgans, M. Probst and O. S. Wolfbeis, J. Chromatogr. B: Anal. Technol. Biomed. Life Sci., 2003, 793, 83-92.

10 I. Bora, S. A. Bogh, M. Santella, M. Rosenberg, T. J. Sørensen and B. W. Laursen, Eur. J. Org. Chem., 2015, 6351-6358.

11 Z. Gao, Y. Hao, M. Zheng and Y. Chen, RSC Adv., 2017, 7, 7604-7609.

12 M. V. Sednev, V. N. Belov and S. W. Hell, Methods Appl. Fluoresc., 2015, 3, 042004.

13 J. R. Mannekutla, B. G. Mulimani and S. R. Inamdar, Spectrochim. Acta, Part A, 2008, 69, 419-426.

14 P. D. McFadden, K. Frederick, L. A. Argüello, Y. Zhang, P. Vandiver, N. Odegaard and D. A. Loy, ACS Appl. Mater. Interfaces, 2017, 9, 10061-10068.

15 M. A. Haidekker and E. A. Theodorakis, Org. Biomol. Chem., 2007, 5, 1669-1678.

16 X. Liu, Z. Xu and J. M. Cole, J. Phys. Chem. C, 2013, 117, 16584-16595.

17 D. Kovacs, X. Lu, L. S. Mészáros, M. Ott, J. Andres and K. E. Borbas, J. Am. Chem. Soc., 2017, 139, 5756-5767.

18 A. Tan, E. Bozkurt and Y. Kara, J. Fluoresc., 2017, 27, 981-992.

19 A. Vyšniauskas, I. López-Duarte, N. Duchemin, T.-T. Vu, Y. Wu, E. M. Budynina, Y. A. Volkova, E. Peña Cabrera, D. E. Ramírez-Ornelas and M. K. Kuimova, Phys. Chem. Chem. Phys., 2017, 19, 25252-25259.

20 Ł. Kielesiński, O. Morawski, Ł. Dobrzycki, A. L. Sobolewski and D. T. Gryko, Chem. - Eur. J., 2017, 23, 9174-9184.

21 G. Signore, R. Nifosì, L. Albertazzi and R. Bizzarri, J. Biomed. Nanotechnol., 2009, 5, 722-729.

22 M. González Pérez, M. Sérgio, A. Manhita, A. T. Caldeira and A. Pereira, unpubl. manuscript.

23 D. Nagaraja, H. S. Geethanjali, N. R. Patil, F. M. Sanningannavar, R. A. Kusanur and R. M. Melavanki, Mapana J. Sci., 2013, 12, 49-68.

24 J. J. Aaron, M. Buna, C. Parkanyi, M. S. Antonious, A. Tine and L. Cisse, J. Fluoresc., 1995, 5, 337-347.

25 A. Alimmari, B. Bozic, D. Mijin, A. Marinkovic, N. Valentic and G. Uscumlic, Arabian J. Chem., 2015, 8, 269-278.

26 R. S. Moog, W. W. Davis, S. G. Ostrowski and G. L. Wilson, Chem. Phys. Lett., 1999, 299, 265-271.

27 R. S. Moog, D. D. Kim, J. J. Oberle and S. G. Ostrowski, J. Phys. Chem. A, 2004, 108, 9294-9301.

28 U. S. Raikar, V. B. Tangod, C. G. Renuka and B. M. Mastiholi, Afr. J. Pure Appl. Chem., 2010, 4, 51-57.

29 T. Molotsky and D. Huppert, J. Phys. Chem. A, 2003, 107, 2769-2780.

30 U. S. Raikar, C. G. Renuka, Y. F. Nadaf, B. G. Mulimani, A. M. Karguppikar and M. K. Soudagar, Spectrochim. Acta, Part A, 2006, 65, 673-677.

31 F. Riedel, A. Oehlke and S. Spange, Z. Anorg. Allg. Chem., 2009, 635, 1335-1340.

32 M. A. Haidekker, T. P. Brady, D. Lichlyter and E. A. Theodorakis, Bioorg. Chem., 2005, 33, 415-425.

33 P. Hrdlovic, J. Donovalova, H. Stankovicova and A. Gaplovsky, Molecules, 2010, 15, 8915-8932.
34 C. Reichardt, Solvents and solvent effects in organic chemistry, Wiley-VCH Verlag GmbH \& Co. KGaA, Weinheim, Printed, Third., 2003.

35 A. Hörner, T. Hagendorn, U. Schepers and S. Bräse, Bioconjugate Chem., 2015, 26, 718-724.

36 C. Reichardt, Chem. Rev., 1994, 94, 2319-2358.

37 S. Yordanova, I. Petkov and S. Stoyanov, J. Chem. Technol. Metall., 2014, 49, 601-609.

38 L. Giordano, V. V. Shvadchak, J. A. Fauerbach, E. A. Jares-Erijman and T. M. Jovin, J. Phys. Chem. Lett., 2012, 3, 1011-1016.

39 M. Tanioka, S. Kamino, A. Muranaka, Y. Shirasaki, Y. Ooyama, M. Ueda, M. Uchiyama, S. Enomoto and D. Sawada, Phys. Chem. Chem. Phys., 2017, 19, 1209-1216.

40 Z. Liu, Y. Wang, X. Pan, Q. Ge, Q. Ma, Q. Li, T. Fu, C. Hu, X. Zhu and J. Pan, Front. Microbiol., 2017, 8, 1-9.

41 N. Panchuk-voloshina, R. P. Haugland, J. Bishop-stewart, M. K. Bhalgat, P. J. Millard, F. Mao, W. Leung, R. P. Haugland, N. P. Voloshina and J. B. Stewart, J. Histochem. Cytochem., 1999, 47, 1179-1188.

42 M. J. Kamlet, J. L. M. Abboud, M. H. Abraham and R. W. Taft, J. Org. Chem., 1983, 48, 2877-2887.

43 J. Catalán, J. Phys. Chem. B, 2009, 113, 5951-5960.

44 A. T. R. Williams, S. A. Winfield and J. N. Miller, Analyst, 1983, 108, 1067-1071.

45 M. J. Frisch, G. W. Trucks, H. B. Schlegel, G. E. Scuseria, M. A. Robb, J. R. Cheeseman, G. Scalmani, V. Barone, G. A. Petersson, H. Nakatsuji, X. Li, M. Caricato, A. V. Marenich, J. Bloino, B. G. Janesko, R. Gomperts, B. Mennucci, J. V. Hratchian, A. F. Ortiz, J. L. Izmaylov, D. Sonnenberg, F. Williams-Young, F. Ding, F. Lipparini, J. Egidi, B. Goings, A. Peng, T. Petrone, T. Henderson, D. Ranasinghe, V. G. Zakrzewski, J. Gao, N. Rega, G. W. Zheng, M. Liang, M. Hada, M. Ehara, K. Toyota, R. Fukuda, J. Hasegawa, M. Ishida, T. Nakajima, Y. Honda, O. Kitao, H. Nakai, T. Vreven, K. Throssell, J. A. Montgomery Jr, J. E. Peralta, F. Ogliaro, M. J. Bearpark, J. J. Heyd, E. N. Brothers, K. N. Kudin, V. N. Staroverov, T. A. Keith, R. Kobayashi, J. Normand, K. Raghavachari, A. P. Rendell, J. C. Burant, S. S. Iyengar, J. Tomasi, M. Cossi, J. M. Millam, M. Klene, C. Adamo, R. Cammi, J. W. Ochterski, R. L. Martin, K. Morokuma, O. Farkas, J. B. Foresman and D. J. Fox, Gaussian 16 Revision B.01, Gaussian, Inc., Wallingford CT, 2016.

46 A. D. Becke, J. Chem. Phys., 1993, 98, 5648-5652.

47 C. Lee, W. Yang and R. G. Parr, Phys. Rev. B: Condens. Matter Mater. Phys., 1988, 37, 785-789.

48 C. Adamo and V. Barone, J. Chem. Phys., 1999, 110, 6158-6170. 49 C. Amovilli, V. Barone, R. Cammi, E. Cancès, M. Cossi, B. Mennucci, C. S. Pomelli and J. Tomasi, Adv. Quantum Chem., 1998, 32, 227-262.

50 M. Cossi, N. Rega, G. Scalmani and V. Barone, J. Chem. Phys., 2001, 114, 5691-5701.

51 P.-F. Loos, J. Preat, A. D. Laurent, C. Michaux, D. Jacquemin, E. A. Perpète and X. Assfeld, J. Chem. Theory Comput., 2008, 4, 637-645.

52 J. Preat, D. Jacquemin, V. Wathelet, J.-M. André and E. A. Perpète, Chem. Phys., 2007, 335, 177-186. 
53 L. Briquet, D. P. Vercauteren, J.-M. André, E. A. Perpète and D. Jacquemin, Chem. Phys. Lett., 2007, 435, 257-262.

54 D. Jacquemin, E. A. Perpète, I. Ciofini and C. Adamo, Acc. Chem. Res., 2009, 42, 326-334.

55 M. Amaro, H. A. L. Filipe, J. P. Prates Ramalho, M. Hof and L. M. S. Loura, Phys. Chem. Chem. Phys., 2016, 18, 7042-7054.
56 J. Preat, P.-F. Loos, X. Assfeld, D. Jacquemin and E. A. Perpète, THEOCHEM, 2007, 808, 85-91.

57 J. Preat, D. Jacquemin, V. Wathelet, J.-M. André and E. A. Perpète, J. Phys. Chem. A, 2006, 110, 8144-8150.

58 M. O. B. Sousa, M. D. Vargas and F. S. Miranda, J. Mol. Struct., 2018, 1164, 260-270. 